\title{
Streptococcal emm types associated with T- agglutination types and the use of conserved emm gene restriction fragment patterns for subtyping group A streptococci
}

\author{
B. BEALL, R. R. FACKLAM, J. A. ELLIOTT, A. R. FRANKLIN, T. HOENES, D. JACKSON, \\ L. LACLAIRE, T. THOMPSON and R. VISWANATHAN
}

Centers for Disease Control and Prevention, Childhood and Respiratory Infections, Atlanta, GA 30333, USA

\begin{abstract}
The T-agglutination types were determined for a diverse collection of 1531 group $A$ streptococci for which the $5^{\prime} \mathrm{M}$ protein gene (emm) sequences had been analysed. The majority of the T-agglutination types correlated with previously seen $\mathrm{M} / \mathrm{emm} / \mathrm{T}$-type associations; however, several new associations were found. Analysis of a subset of this collection - which included 1157 clinical isolates with multiply encountered $\mathrm{emm}$ types - found that $\mathrm{emm}$ amplicon restriction profiles of isolates sharing identical $T$ types and opacity factor phenotypes are useful for detecting groups of isolates with identical $\mathrm{emm}$ genes. Many emm genes of known $5^{\prime}$ sequence display a highly conserved restriction pattern amongst clinical isolates widely separated both geographically and temporally.
\end{abstract}

\section{Introduction}

Subtyping of clinical isolates of group A streptococci (GAS) has relied historically upon serotyping of the surface $M$ protein with polyclonal antisera. However, there are many problems inherent to $M$ typing, including the large number of $\mathrm{M}$ serotypes and the lack of availability of comprehensive $M$ typing sera sets. Currently, there are c. 80 recognised $\mathrm{M}$ serotypes, 15 provisional $M$ types, and many more deduced new specificities on the basis of emm gene sequences that encode the hypervariable regions of $\mathrm{M}$ proteins. A recent study showed that rapid $\mathrm{emm}$ variable region sequence analysis (emm typing) of GAS in combination with conventional T-agglutination pattern determination and opacity factor (OF) phenotype determination, is a useful means by which to subtype this organism. This system is almost $100 \%$ efficient and often deduces the $M$ serotype [1, 2]. This facet of emm typing is especially important because $\mathrm{emm}$ gene sequences correlated with known serospecificities are accessible from the GenBank and for the most part complement $c$. 50 years of accumulated $M$ serotyping data [1-10]. The present study shows the T-agglutination types associated with the various emm types that were encountered over the last 3 years. Furthermore, an

Received 8 Oct. 1997; accepted 2 Feb. 1998.

Corresponding author: Dr B. Beall. improvement of the emm typing system that takes advantage of emm type specific conserved restriction patterns is clearly demonstrated.

\section{Materials and methods}

\section{Strains}

Approximately $64 \%$ of the 1531 strains for which Tagglutination patterns were determined represented the total number of sterile site isolates obtained from five regions within the USA for $1-3$ years during the period 1994-1997. These regions included metropolitan Atlanta, GA; metropolitan San Francisco, CA; metropolitan Minneapolis, $\mathrm{MN}$; Connecticut state and Oregon state. Approximately $13 \%$ of the strains represented sporadic sterile site isolates from other regions within the USA. The remaining strains were obtained from various sources outside the USA including Bulgaria, Poland, Malaysia, Korea, Australia, New Zealand and South America. Approximately 100 strains were $M$ type and $M$ provisional type $C D C$ reference strains.

\section{Subtyping procedures}

$\mathrm{T}$ typing and $\mathrm{OF}$ determination were performed as described previously $[8,11]$; emm-specific PCR products were prepared and the $5^{\prime}$ end of $\mathrm{emm}$ genes were sequenced for type determination as described pre- 
viously [2]. For generation of an emm amplicon restriction profile (ERP), $8.5 \mu \mathrm{l}$ of unpurified PCR product were digested in a total volume of $10 \mu \mathrm{l}$ containing $1 \times$ reaction buffer 4 (Gibco-BRL), 5 units of Hae III and 5 units of Hinc II. The samples were incubated for $1 \mathrm{~h}$ (or longer) at $37^{\circ} \mathrm{C}$, electrophoresed in agarose $2 \% \mathrm{w} / \mathrm{v}$ gels containing ethidium bromide $1 \mu \mathrm{g} / \mathrm{ml}$ in TAE buffer (40 mM Tris-acetate, $\mathrm{pH} 8.2$, $20 \mathrm{mM}$ sodium acetate, $1 \mathrm{mM}$ EDTA) at $100 \mathrm{~V}$ for $30 \mathrm{~min}$, visualised on a UV transilluminator and photographed.

Sequences were given the indicated GenBank emm type designations if $\geqslant 95 \%$ identical over at least the first 160 bases, permitting one in-frame deletion or insertion of up to seven codons or one frameshift of up to seven codons. Such derivatives made up $<3 \%$ of the total sequences represented. GenBank accession numbers for most of the emm type sequences are given in references $[1,2,5,9,10,12,13$ and 14] except for the emm sequences found from our M67, M68, and M69 reference strains (emm67, emm68, and emm69 in Table 1) that differed from the data described by Whatmore and colleagues [10]. Our M65 and M69 reference strains have the same $5^{\prime} \mathrm{emm}$ sequence, which was identical to the previously determined emm65 sequence [10]. It has also been determined that M44 and M61 strains have the same $5^{\prime} \mathrm{emm}$ sequence $[1,10]$. The sequences shown above that are not listed in these references were obtained in this laboratory and the accession numbers are as follows: $\quad$ emm67 $=\mathrm{AF} 025949, \quad$ emm68 $=\mathrm{AF} 025948$,
ST2034 $=$ U74320, $\quad$ PT2631 $=$ U94589, $\quad$ ST4935 $=$ $\mathrm{U} 92492$, emm $70=\mathrm{AF} 035838$.

\section{Results}

T-agglutination patterns seen in different emm types

Table 1 lists the $\mathrm{T}$ patterns and OF reactions that have been determined for $90 \mathrm{emm}$ types. Data are not given for the 25 new emm types that were found only amongst sporadic isolates. The majority (1244 of 1363 T-typable isolates) of the $\mathrm{T}$ agglutination patterns shown in Table 1 correlated with previously published $\mathrm{M}$ type/T type/OF associations [1-5], but there were also many unpublished associations (Table 1). Nearly $12 \%$ of the isolates $(178 / 1531)$ were found to be $\mathrm{T}$ non-typable.

\section{emm specific amplicon restriction profiling}

Table 2 shows that 945 of 1157 geographically diverse clinical isolates had a predominant ERP type shared within their respective emm sequence types. These isolates were collected primarily from normally sterile sites, although c. $2 \%$ of them were isolated from throat or skin lesion cultures. These isolates represented 32 different $\mathrm{emm}$ sequence types that we have encountered on multiple occasions. For each of the emm types with the exception of emm6, emm 76 and ST2974, there was a single restriction pattern accounting for $>60 \%$ of isolates within that emm type; however, for some of the

Table 1. emm types associated with T-agglutination patterns and opacity factor (OF) reaction

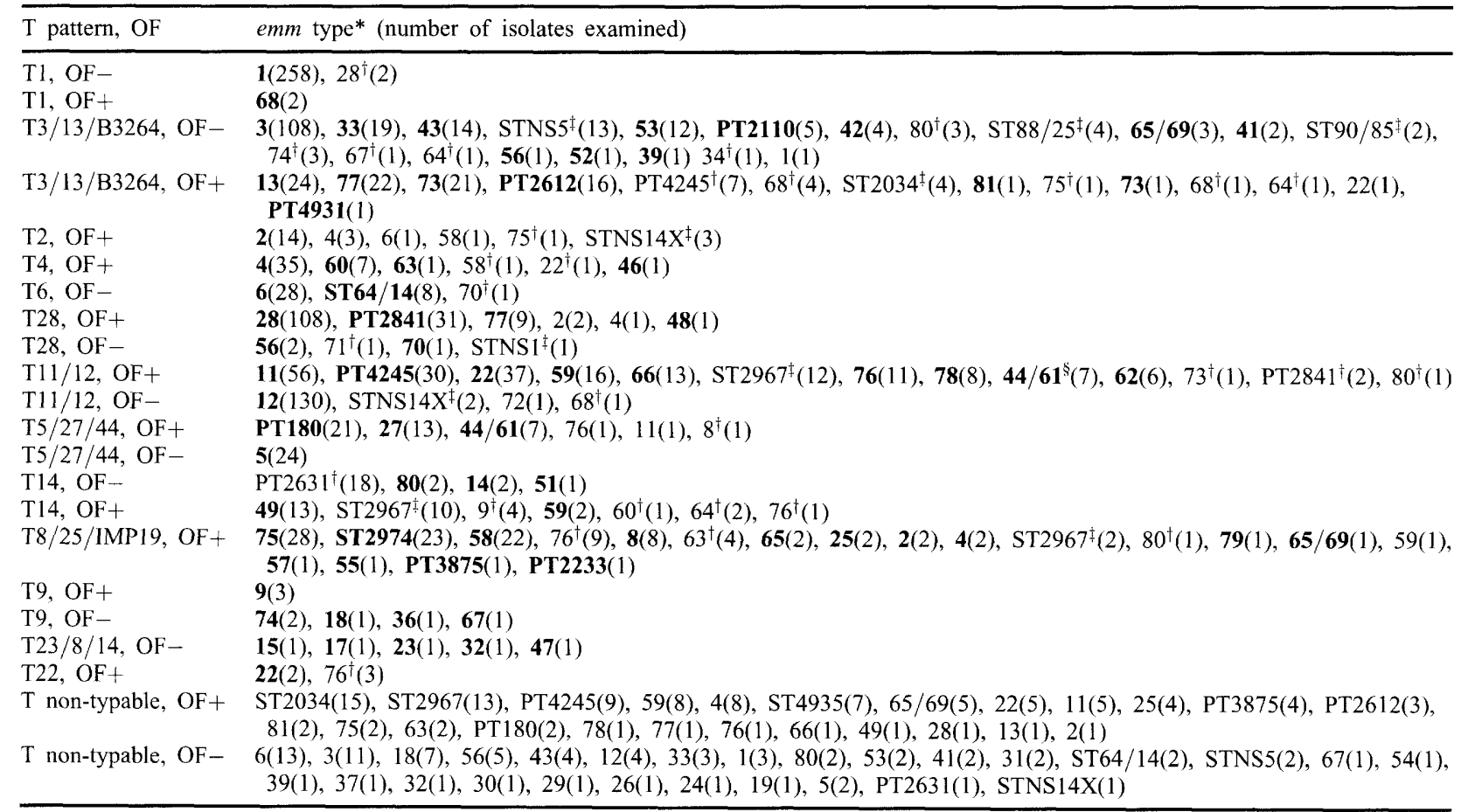

* emm types expected for the $\mathrm{T}$ pattern and $\mathrm{OF}$ reaction are in bold.

${ }^{\dagger}$ Represents an unpublished $\mathrm{M}(\mathrm{emm}) / \mathrm{T}$-agglutination pattern association.

${ }_{\ddagger}^{\ddagger} \mathrm{M}(\mathrm{emm})$ type/T-agglutination pattern associations have not been previously made for this emm type. 
Table 2. Major emm amplicon restriction profiles shared among isolates of the same emm sequence type

\begin{tabular}{|c|c|c|c|}
\hline emm type & $\begin{array}{l}\text { Number }(\%) \\
\text { of isolates }\end{array}$ & $\begin{array}{l}\text { Number }(\%) \\
\text { from the USA }\end{array}$ & Number $(\%)$ from other countries \\
\hline emml & $225 \quad(99)$ & $198 \quad(88)$ & 27 (12): S. America \\
\hline emm2 & $17(100)$ & $12(71)$ & 5 (29): S. America, Bulgaria \\
\hline emm3 & $104(98)$ & $81 \quad(78)$ & 23 (22): S. America, Korea, Bulgaria \\
\hline emm4 & 34 (79) & 27 (79) & 7 (21): S. America, Poland, Malaysia \\
\hline emm5 & $14(61)$ & $13(93)$ & (7): Malaysia \\
\hline emm6 & $17 \quad(50)$ & $9 \quad(53)$ & 8 (47): S. America, Korea, Poland \\
\hline emm11 & $34 \quad(87)$ & 33 (97) & 1 (3): S. America \\
\hline emm12 & $120(100)$ & $95 \quad(79)$ & $\begin{array}{l}25 \text { (21): S. America, Bulgaria, } \\
\text { Malaysia }\end{array}$ \\
\hline emml3 & $10(83)$ & $6(60)$ & 4 (40): S. America \\
\hline emml8 & $6(100)$ & & \\
\hline emm 22 & 28 (93) & $19(68)$ & 9 (32): S. America \\
\hline emm 28 & $94 \quad(97)$ & $89(95)$ & 5 (5): Korea, Poland \\
\hline emm 33 & $28(100)$ & 27 (96) & 1 (4): Korea \\
\hline emm4l & $4(67)$ & $4(100)$ & \\
\hline emm43 & $12(100)$ & $12(100)$ & \\
\hline emm44/61 & $7(100)$ & $7(100)$ & \\
\hline emm49 & $5(100)$ & $4(80)$ & 1 (20): S. America \\
\hline emm53 & $7(100)$ & $6 \quad(86)$ & 1 (14): New Zealand \\
\hline emm 58 & $11(65)$ & $11(100)$ & \\
\hline emm59 & $17(100)$ & $16(88)$ & 1 (12): S. America \\
\hline emm65/69 & $8(100)$ & & 8 (100): Bulgaria, S. America \\
\hline emm66 & $8(100)$ & $8(100)$ & \\
\hline emm 73 & $4(100)$ & $4(100)$ & \\
\hline emm 75 & $18(100)$ & $12(67)$ & 6 (33): Korea, S. America \\
\hline emm76 & $7 \quad(34)$ & $7(100)$ & \\
\hline emm 77 & $33(97)$ & $26(79)$ & 7 (21): S. America \\
\hline PT4245 & $35(100)$ & $34(97)$ & 1 (3): S. America \\
\hline PT2841 & $17(74)$ & $1 \quad(6)$ & 16 (94): S. America \\
\hline PT2110 & $3(100)$ & $3(100)$ & \\
\hline PT180 & $10(83)$ & $6(60)$ & 4 (40): S. America, Malaysia \\
\hline 2974-1 & $8 \quad(57)$ & $5 \quad(62)$ & 3 (38): S. America \\
\hline
\end{tabular}

*Percentage of isolates with the most frequently occurring emm restriction profile for the indicated emm type.

emm types the results could be biased because of small sample size or geographic distribution. Twenty-four of these major profiles are shown in Fig. 1 (lanes 2, 3, 514, 16-29), and for emm6 two secondary ERPs are shown (Fig. 1, lanes 1 and 4). As the emm gene amplicons of different types vary extensively in size ( $c$. 900-1600 bp) and usually have two or more Hae II or Hinc II sites, or both, in most cases major restriction profiles representing one emm type are readily distinguishable from the major ERP of other emm types.

A minority of emm genes lacked both Hinc II and Hae III sites (lanes 12, 19 and 21 in Fig. 1); they were distinguishable from other $\mathrm{emm}$ genes lacking these sites by differing sizes (Fig. 1; compare amplicons for emm33 and emm43 in lanes 19 and 21 with emm66 amplicon in lane 12) or by unique restriction profiles generated by other frequent cutters such as $R s a \mathrm{I}$ or Dpn II. Although the emm33 and emm43 PCR fragments lack Hinc II and Hae III sites, and were almost of identical size (Fig. 1, lanes 19 and 21), the two PCR fragments were readily distinguishable by Rsa I cleavage profiles. Regardless of amplicon sizes, alternate restriction enzymes are always used for amplicons lacking Hinc II and Hae III sites.
Currently, for GAS subtyping ERPs are generated in association with emm sequence results at this laboratory as follows; isolates are subjected to $T$ antigen typing and OF determination as described previously $[8,11]$. Those isolates from the same general population area during a 1-year time period that have related T-antigen patterns, the same OF phenotype and identical ERP are identified. A random sample of emm amplicons from such a group is subjected to $\mathrm{emm}$ variable region sequence analysis, which effectively determines the emm type of the entire group. A false deduced emm designation result has not been found with this scheme.

An example of the routine use of ERP comparison to reduce the numbers of samples sequenced is shown in Fig. 2. Lanes 2-10 represent the ERPs of nine T type 28 , OF + Brazilian isolates. Lanes 2 and 5-10 are the most common emmPT2841 ERP (compare with Fig. 1, lane 8). emm sequence analysis of two of these isolates showed almost $100 \%$ identity to the emm PT2841 gene. Lane 3 had a different ERP; however, this PCR fragment was also found to have the emmPT2841 sequence. Comparison of these two emmPT2841 ERPs indicated the probable loss of one Hinc II or Hae III site in the amplicon shown in 


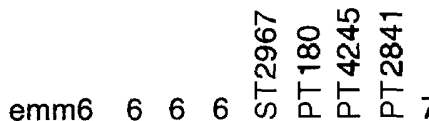

emm6 $6 \quad 6 \quad 6 \quad$ 心

123456789101112131415161718192021222324252627282930
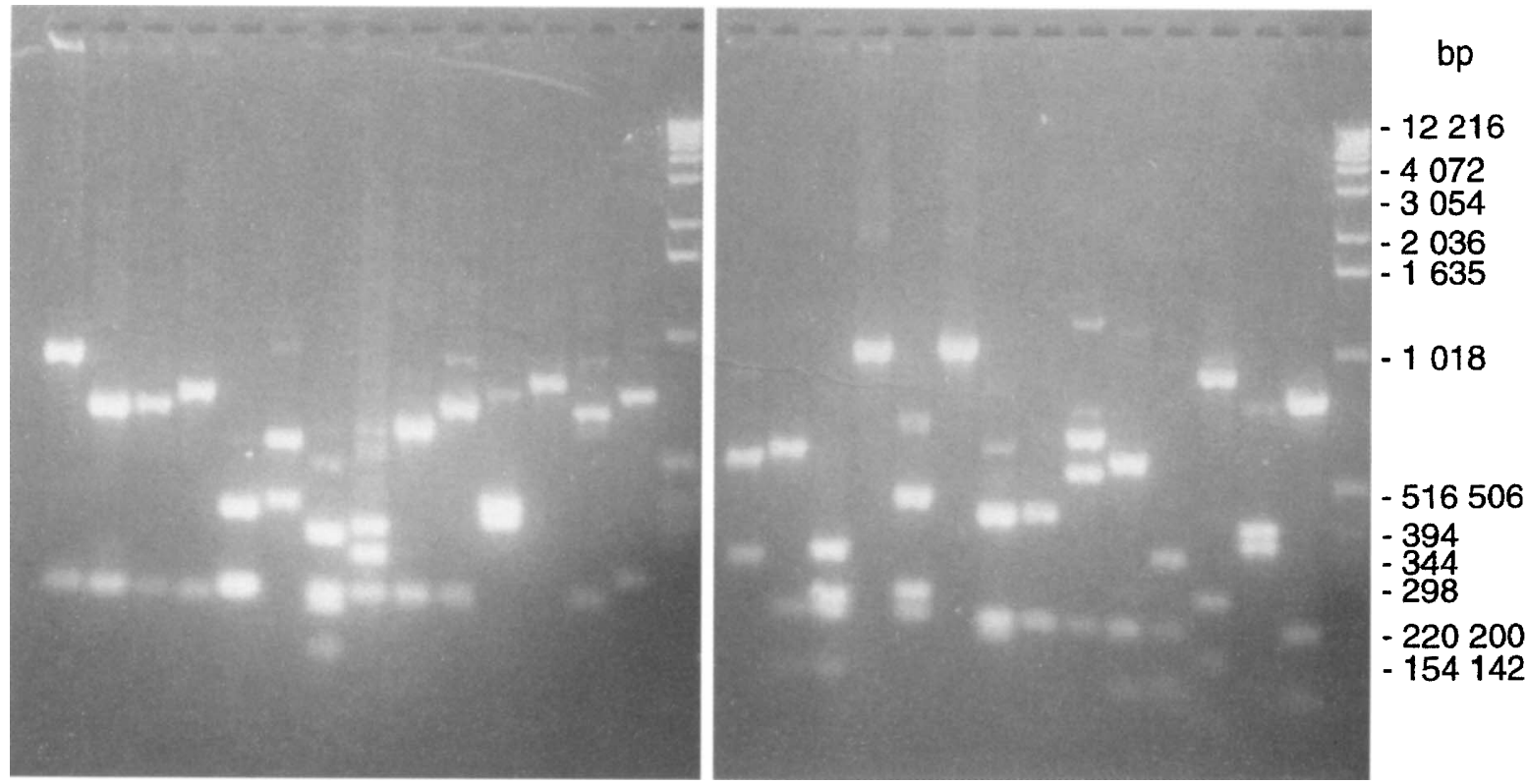

Fig. 1. Frequent $\mathrm{emm}$ amplicon restriction profiles (Hinc II and Hae III double digest profiles) of 25 different $\mathrm{emm}$ types. Lanes 1-4, depict three emm6 profiles; 5-14, the most frequently encountered emm amplicon restriction profiles for the indicated emm types; 15 and 30, Gibco-BRL kb ladder size standard.

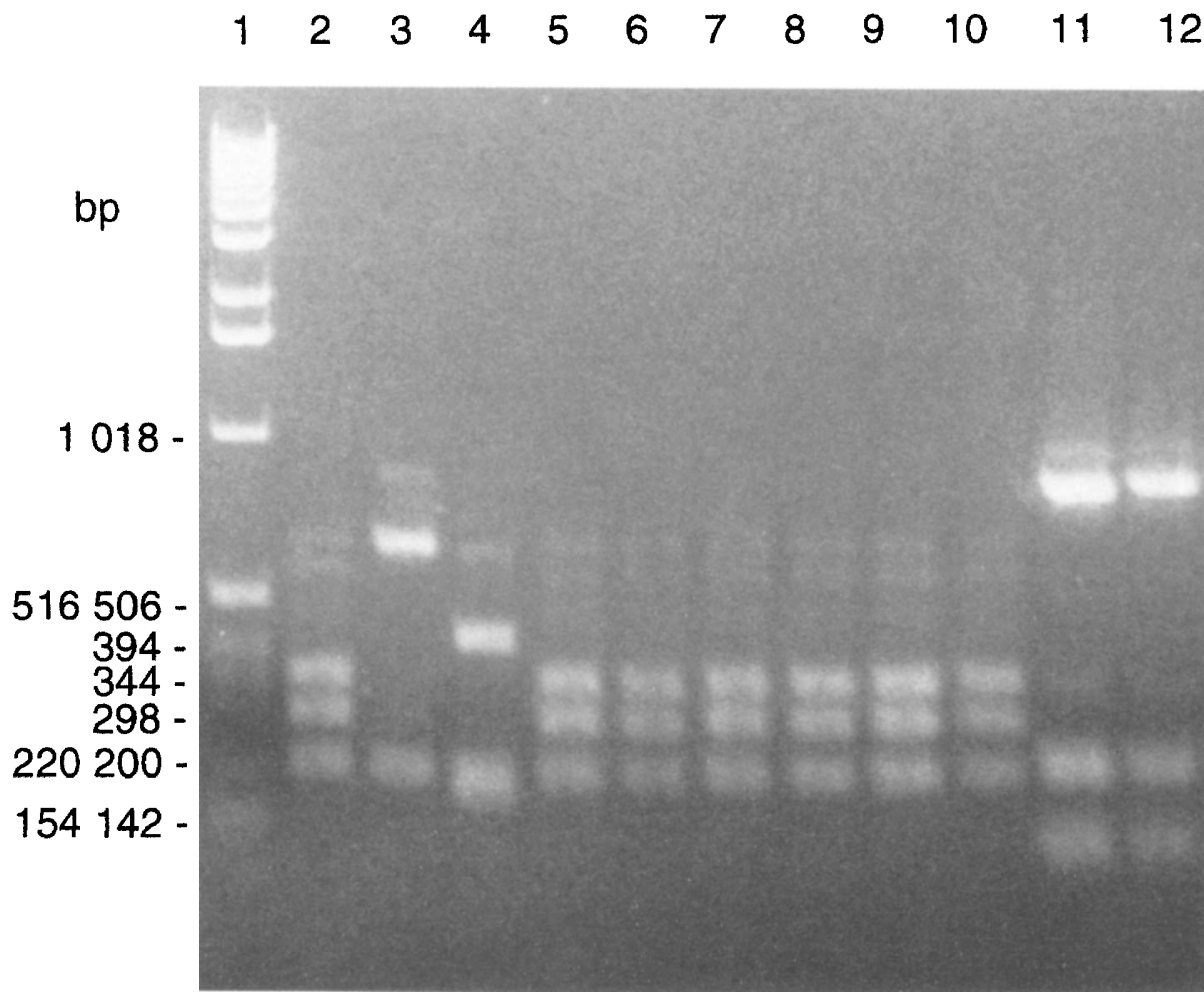

Fig. 2. ERP analysis of 11 Brazilian GAS isolates. Lanes 2-10, were obtained from nine independent T28 OF+ isolates; 11 and 12, were obtained from two independent T1 OF- isolates. Selected uncut samples were subjected to sequence analysis and found to be PT2841 (lanes 2, 3 and 5), emm28 (4) and emml (11).

lane 3. One of the $\mathrm{T} 28 \mathrm{OF}+$ isolates produced the most common emm28 ERP (compare Fig 2, lane 4 with Fig. 1, lane 22) and sequence analysis revealed near identity with emm28 (not shown). Also shown in Fig. 2 is the major emml ERP (Fig. 1, lanes 11 and 12) shared by two independent $T$ type 1, OF- 
isolates. Sequence analysis of one of the isolates revealed identity to the emml sequence.

ERP analysis is useful for deducing the emm type for epidemiologically related sets of isolates that contain variant alleles of previously characterised emm genes. For example, two emm3 isolates with ERP profiles not typical of the major emm 3 ERP were four codon deletion derivatives of emm3. In addition, on numerous occasions ERP analysis has been used for the identification of epidemiologically related sets of isolates containing emm genes that have not been encountered previously. For such isolates, that have related $\mathrm{T}$ patterns, identical $\mathrm{OF}$ phenotypes and identical ERP, it is essential to examine the $5^{\prime}$ variable emm sequence of only one of the isolates to predict the emm type of the whole group (unpublished data).

\section{Discussion}

The sample set in the present study was biased in that primarily isolates from the USA were analysed. Even so, it is interesting that relatively few new emm sequence types have been encountered. The definition of an $\mathrm{emm}$ type is given here as $\geqslant 95 \%$ identical over the first 160 bases of sequence obtained with primer emmseq2 [2], permitting one in-frame deletion or insertion of up to seven codons or one frameshift of up to seven codons. The majority of the known emm types are only $50-85 \%$ identical over the first 160 bases obtained. The basis of the relative rarity of 'intermediate' emm types with $85-95 \%$ sequence identity has not yet been determined.

For most of the emm genes analysed the study found a striking lack of allelic diversity determined by sequence and ERP analysis from geographically distinct isolates (Table 1). These results may indicate that these are evolutionarily stable $\mathrm{emm}$ genes that infrequently undergo genetic variation. In contrast, for emm genes such as emm6, emm76 and ST2974 multiple alleles of different sizes were encountered (three of emm6 are shown in Fig. 1, lanes 1-4), at least partially due to different patterns of homologous recombination between direct repeats in the hypervariable region [15, unpublished observations].

For some isolates with the same emm types and ERPs more than one $T$ agglutination pattern is encountered. We have found no correlation between different ERP subtypes within a given emm type with differences in $\mathrm{T}$ types. Generally, we have found that pulsed-field gel electrophoresis patterns of chromosomal digests reflect that isolates of the same emm type, but with different $\mathrm{T}$ types, are genetically divergent (unpublished observations), whilst isolates with identical $\mathrm{T}$ and emm types are more closely related. The compilation of the varied emm/T-antigen pattern associations shown in
Table 1 is an indication of the genetic diversity within emm types that is due to horizontal genetic transfer events between unrelated strains.

The results shown here, which show major conserved emm restriction fragment patterns for $32 \mathrm{emm}$ types, are analogous to those found previously when Hae III was used to find characteristic emm restriction profiles within three different GAS serotypes [16]. Furthermore, outbreak-related strains could be distinguished from other strains within the same M-type by the use of characteristic Hae III profiles [16]. We have also found this strategy useful for this purpose (unpublished data); however, the combination of Hae III and Hinc II often provides more discriminatory electrophoretic profiles than that of Hae III alone.

For many emm types there is a high probability of deducing emm types from geographically diverse isolates by their ERP alone in combination with $T$ typing and $\mathrm{OF}$ determination. For example, this laboratory has rarely encountered alternate ERPs for the distinctive patterns seen for the commonly encountered emml and emm 3 types, and has not found an alternative ERP for the frequently occurring emm12 isolates (Table 1 and Fig. 1, lanes 24, 27 and 29). For commonly encountered ERP profiles, emm types can be detected by concurrently electrophoresing digest standards or comparison with saved electrophoretic patterns on a computer scanner.

We thank the Emerging Infections Programs (EIP), supported by the National Center for Infectious Diseases for isolates resulting from active surveillance; California EIP: A. Reingold, Gretchen Rothrock and D. Vugia; Connecticut EIP: M. Cartter, J. Hadler, Patricia Mshar, C. Morin and Nancy Barrett; Georgia EIP: Wendy Baughman and Monica Farley; Minnesota EIP: Jean Rainbow, Kristine MacDonald and M. Osterholm; Oregon EIP: S. Ladd-WIlson, Karen Stefonek, P. Cieslak; and Katherine O'Brien for isolates from sporadic cases of necrotising fasciitis.

\section{References}

1. Beall B, Facklam R, Thompson T. Sequencing emm-specific PCR products for routine and accurate typing of group A streptococci. J Clin Microbiol 1995; 34: 953-958.

2. Beall B, Facklam R, Hoenes T, Schwartz B. A survey of emm gene sequences and T-antigen types from systemic Streptococcus pyogenes infection isolates collected in San Francisco, California; Atlanta, Georgia, and Connecticut in 1994 and 1995. J Clin Microbiol 1997; 35: 1231-1235.

3. Colman G, Tanna A, Efstratiou A, Gaworzewska ET. The serotypes of Streptococcus pyogenes present in Britain during 1980-1990 and their association with disease. J Med Microbiol 1993; 39: $165-178$.

4. Fraser CAM, Colman G. Some provisional M-types among Streptococcus pyogenes (Lancefield group A). In: Kimura Y, Kotami S, Shiokawa Y (eds) Recent advances in streptococci and streptococcal diseases. Bracknell, Reedbooks. 1985: 35-36.

5. Johnson DR, Kaplan EL. A review of the correlation of Tagglutination patterns and M-protein typing and opacity factor production in the identification of group A streptococci. $J$ Med Microbiol 1993; 38: $311-315$.

6. Kaufhold A, Podbielski A, Baumgarten G, Tap J, Blokpoel M, Schouls L. Rapid typing of group A streptococci by the use of DNA amplification and non-radioactive allele-specific oligonucleotide probes. FEMS Microbiol Lett 1994; 119: 19-26. 
7. Lancefield RC. Current knowledge of the type-specific M antigens of group A streptococci. J Immunol 1962; 89: 307-313.

8. Moody MD, Padula J, Lizana D, Hall CT. Epidemiologic characterization of group A streptococci by T-agglutination and M-precipitation tests in the public health laboratory. Health Lab Sci 1996; 2: 149-162.

9. Podbielski A, Melzer B, Lutticken E. Application of the polymerase chain reaction to study the $M$ protein(-like) gene family in beta-hemolytic streptococci. Med Microbiol Immunol 1991; 180: 213-227.

10. Whatmore AM, Kapur V, Sullivan DJ, Musser JM, Kehoe MA Non-congruent relationships between variation in emm gene sequences and the population genetic structure of group A streptococci. Mol Microbiol 1994; 14: 619-631.

11. Maxted WR, Widdowson JP, Fraser CAM, Ball LC, Bassett DCJ. The use of the serum opacity reaction in the typing of group-A streptococci. J Med Microbiol 1973; 6: 83-90.

12. Pack TD, Podbielski A, Boyle MDP. Identification of an amino acid signature sequence predictive of protein G-inhibitable $\mathrm{IgG}_{3}$-binding activity in group-A streptococcal IgG-binding proteins. Gene 1996; 171: 65-70.

13. Relf WA, Martin DR, Sriprakash KS. Identification of sequence types among the M-nontypeable group A streptococci. J Clin Microbiol 1992; 30: 3190-3194.

14. Relf WA, Martin DR, Sriprakash KS. Antigenic diversity within a family of $M$ proteins from group A streptococci: evidence for the role of frameshift and compensatory mutations. Gene 1994; 144: 25-30.

15. Hollingshead SK, Fischetti VA, Scott JR. Size variation in group A streptococcal $\mathrm{M}$ protein is generated by homologous recombination between intragenic repeats. Mol Gen Genet 1987; 207: 196-203.

16. Stanley J, Desai M, Xerry J, Tanna A, Efstratiou A, George R. High-resolution genotyping elucidates the epidemiology of group A Streptococcus outbreaks. J Infect Dis 1996; 174: 500-506. 